\title{
Implementing NICE guidance
}

\author{
Jayne Chidgey ${ }^{1} \quad$ Gillian Leng ${ }^{2}$ Terence Lacey ${ }^{3}$
}

J R Soc Med 2007; 100:448-452

\section{INTRODUCTION}

The National Institute for Health and Clinical Excellence (NICE) was established as a Special Health Authority in April 1999 to promote clinical excellence and the effective use of resources within the NHS. NICE is the independent organization responsible for providing national guidance on the promotion of good health and the prevention and treatment of ill health (Box 1). This article highlights the robust methodology used to develop NICE guidance and NICE's approach to economic analysis, the mechanisms by which the clinical community is involved in guidance development, the practical support available to aid implementation of guidance, and the advantages to doctors (and other clinicians) of using NICE guidance.

\section{WHY USE NICE GUIDANCE?}

NICE guidance can help doctors by:

- Ensuring care provided is based on the best evidence available;

- Effectively targeting resources and efforts at the areas that offer the most significant health improvement;

- Ensuring clinicians meet the standards set by regulatory bodies and that they consider NICE guidance when exercising their clinical judgement;

- Enabling staff dealing with patient queries to have confidence in the approaches to care;

- Providing up to date reviews of new evidence.

One of the core duties of a doctor is to maintain good medical practice by keeping up to date. ${ }^{1}$ But with more than two million clinical research articles published each year, ${ }^{2}$ 'keeping up to date' is not easy. The development and use of clinical guidance based on assessment of clinical and cost effectiveness is probably the most important approach to ensuring patients derive the full benefits from clinical research.

NICE's clinical guideline programme is arguably the largest in the world, and is unique in considering both cost and clinical effectiveness. Nearly 50 clinical guidelines have

${ }^{1}$ Implementation Consultant, ${ }^{2}$ Implementation Systems Director and ${ }^{3}$ Data Analyst, National Institute for Health and Clinical Excellence, MidCity Place, 71 High Holborn, London WC1V 6NA

Correspondence to: Dr Jane Chidgey

Email: jayne.chidgey@nice.org.uk

\section{BoX 1 NICE guidance areas}

- Health technologies: guidance on the use of new and existing medicines, treatments and procedures within the NHS (technology appraisals and interventional procedure guidance)

- Clinical practice: guidance on the appropriate treatment and care of people with specific diseases and conditions within the NHS (clinical guidelines)

- Public health: guidance on the promotion of good health and the prevention of ill health for those working in the NHS, local authorities and the wider public and voluntary sector (public health intervention guidance and public health programme guidance)

now been published, and over 30 are currently in production. Similarly, the health technology appraisal system is internationally recognized for its robust and transparent mechanisms; 98 appraisals have been published to date and 56 are in production. More recently, NICE has started issuing public health guidance (five published to date and 15 in development).

\section{NICE GUIDANCE DEVELOPMENT PROCESSES}

Clinician confidence in the process of developing national guidance - and in the resulting final recommendations - is essential if guidance is going to inform clinical practice and be used. Professionals (and the public) can have confidence in NICE guidance, as the development processes for all types of guidance are underpinned by the key NICE principles of basing recommendations on the best available evidence and involving all stakeholders in a transparent and collaborative manner. In addition, NICE guidance development processes are designed to be rigorous and robust, including the use of independent committees; precise definition of the scope of the guidance; independent and comprehensive assessment of the evidence base, the clarity of presentation and the applicability of the finished product; editorial independence; and consideration of implementation issues. A survey sponsored by the World Health Organization in 2005 scored NICE's guideline on schizophrenia higher than 26 other international guidelines on the same topic. ${ }^{3}$

\section{INVOLVING CLINICIANS}

Clinical support is a key factor in the implementation of NICE guidance. ${ }^{4}$ Obtaining the genuine involvement of 
stakeholders is a challenge for any programme producing national guidance, but support from relevant professional groups is essential if the recommendations are to be accepted and stimulate a change in practice. Clinicians are key stakeholders in all NICE guidance. Important steps in the NICE guidance development process that aim to generate stakeholder support include involvement in the topic selection process; the inclusion of relevant professionals on the guideline development groups and appraisal panels; open and genuine consultation which often results in significant changes to the draft recommendations; and support from the professional colleges and associations. These relationships are reinforced through the National Collaborating Centres, funded by NICE to produce guidelines and which are largely hosted within the Royal Colleges, and the Technology Appraisal Research (TAR) teams which are hosted by universities across the UK.

\section{CONSIDERING CLINICAL AND COST EFFECTIVENESS}

As well as having confidence in the guideline development process and appraisal of the evidence, it is important for clinicians to understand NICE's approach to economic evaluation. In a health-care system with finite resources, such as the NHS, clinical effectiveness on its own is insufficient to form a basis for producing guidance. Therefore NICE bases its recommendations on an assessment of both clinical and cost effectiveness, both in its technology appraisals and in its clinical and public health guidelines.

In cases where two or more clinical management options are being compared, where there is therapeutic equivalence the cheaper option is preferable. However, decisions are often more complex: for example, the option being assessed has better outcomes than current standard practice but costs more. NICE's role when producing its recommendations thus includes an assessment of what increase in health, compared with standard practice, is likely to be gained from the increase in costs. The assessment of this incremental cost effectiveness ratio can be carried out in different ways; NICE's preferred measure is the cost per quality adjusted life year (QALY) — although alternatives are used in circumstances where appropriate data on quality of life are not available (e.g. life years gained, cases averted, or disease-specific outcomes ${ }^{5,6}$ ). Figure 1 demonstrates a plot of length of life (in years) against quality of life (or 'utility' on a $0-1$ scale), comparing the expected profile for a group of patients with some given treatment and others not given treatment (or given a standard treatment versus a new treatment). The initial decline in health seen in the group receiving treatment relates to side effects of the intervention but, in this

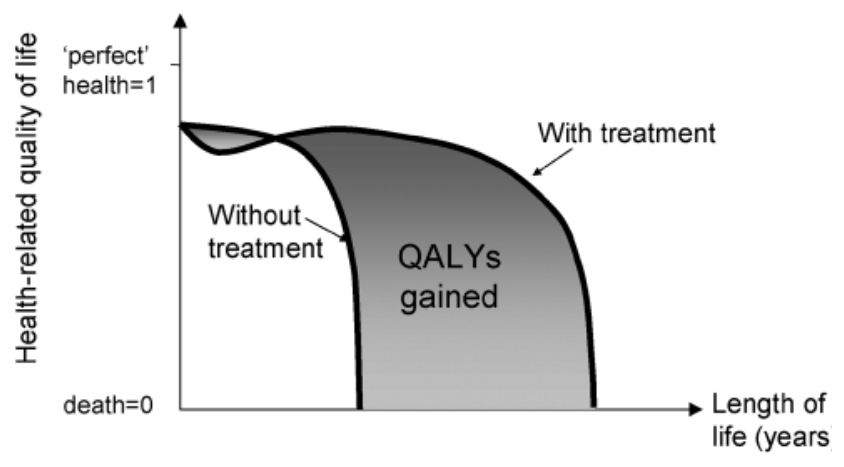

Figure 1 The QALY concept

example, in the longer term both length of life and quality of life are improved in those on treatment.

The area between the two curves is the number of QALYs gained (or lost) for a given treatment. As part of the economic appraisal carried out by NICE, the cost per QALY gained is calculated.

NICE makes its decisions on a case-by-case basis rather than using an absolute threshold when making recommendations for the NHS, although the probability of rejection increases as cost per QALY goes up (Figure 2). The final decision is based on careful deliberation within independent advisory committees and guidance development groups, taking into account a range of factors such as equity, practicalities of implementation, legal and policy constraints, and the extent of uncertainty.

\section{IS NICE GUIDANCE CHANGING CLINICAL PRACTICE?}

There is evidence from the uptake of drugs that guidance from NICE has a significant impact on practice. Figure 3, for example, demonstrates the use of obesity drugs before and after publication of relevant NICE guidance recommending use of the drugs in certain circumstances. Similarly, Figure 4 demonstrates decline in use of the drug

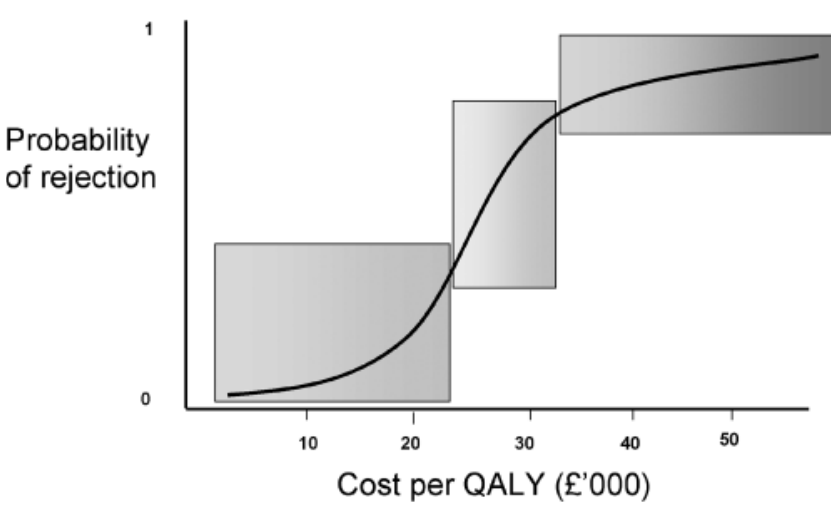

Figure 2 Assessing cost effectiveness 


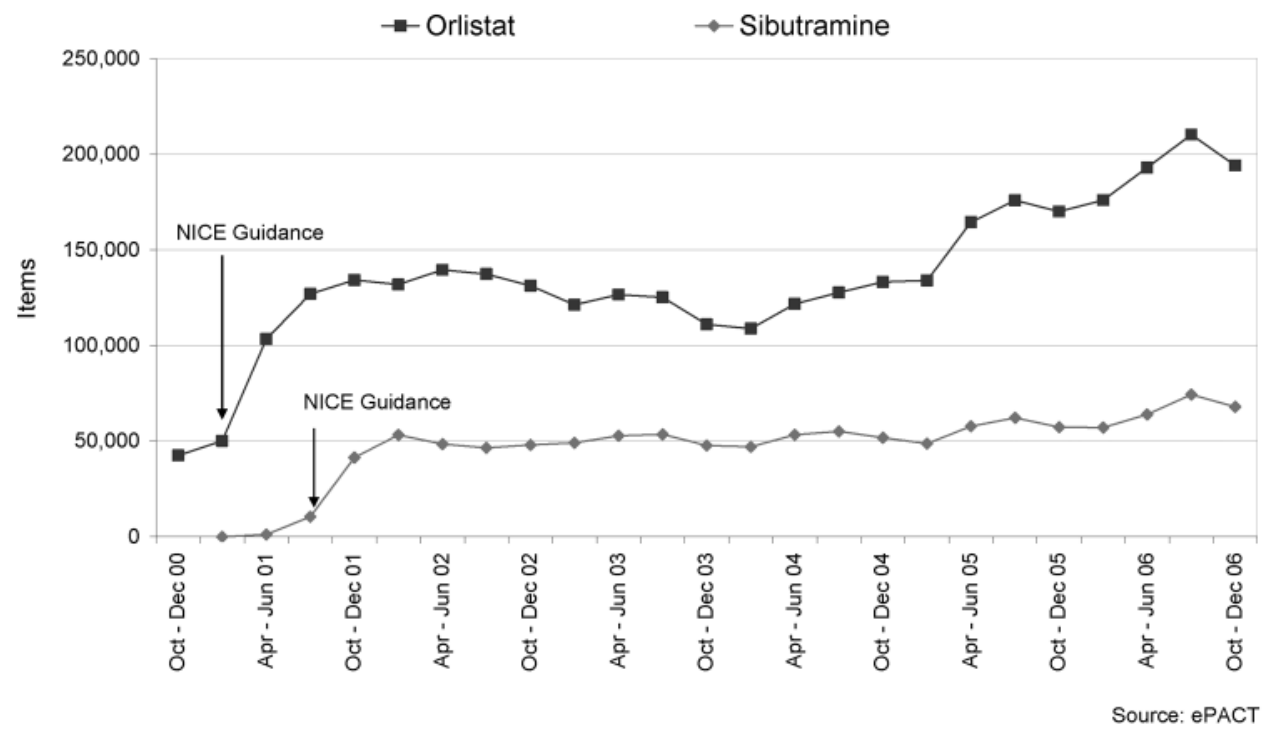

Figure 3 Trends in prescribing volume for orlistat and sibutramine in primary care in England. PACT: This information comes from the electronic Prescribing Analysis and Cost Tool (ePACT) system, which covers prescriptions prescribed by GPs and other non-medical prescribers in England and dispensed in the community in the UK. The Prescription Pricing Division of the NHS Business Services Authority maintains the system. PACT data are used widely in the NHS to monitor prescribing at a local and a national level. Prescriptions written in hospitals that are dispensed in the community (FP10 [HP]) are not included in PACT data. Prescriptions dispensed in hospitals or mental health units, and private prescriptions, are not included in PACT data

anakinra following recommendations of its lack of clinical and cost effectiveness by NICE.

Clear progress is therefore being made on the implementation of NICE technology appraisals, but the implementation of clinical guidelines remains more challenging because of the complexity involved. A study in the UK identified that only $40 \%$ of patients were receiving care in line with best practice guidelines covering four common conditions. ${ }^{7}$ It is recognized that each individual guideline is likely to impact on a range of professionals and

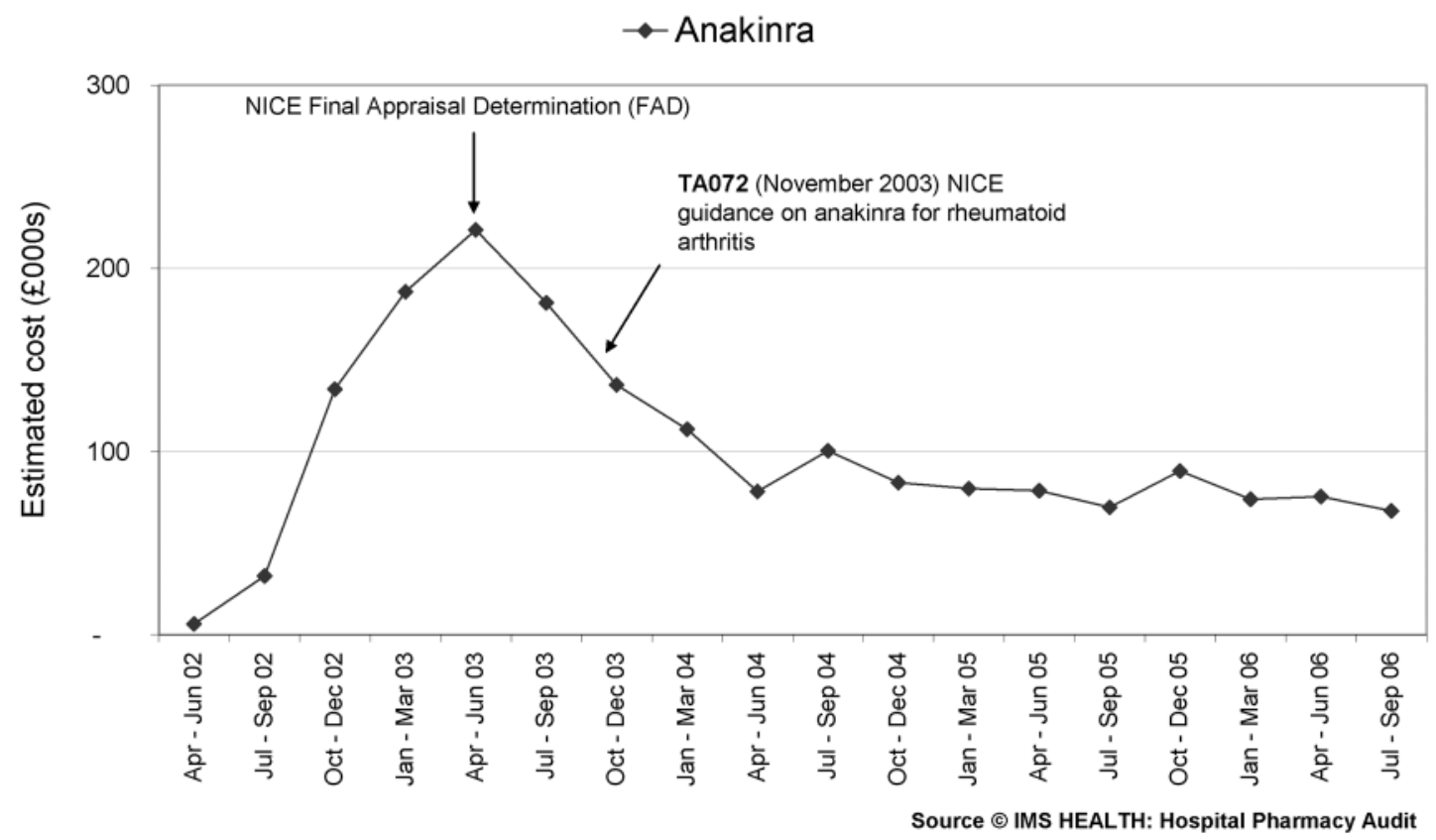

Figure 4 Trends in prescribing cost for anakinra in hospitals in England. IMS HEALTH Hospital Pharmacy Audit: IMS HEALTH collects information from pharmacies in hospital trusts in the UK. The section of this database relating to England is available for monitoring the overall usage in drugs appraised by NICE. The IMS HPAI database is based on issues of medicines recorded on hospital pharmacy systems. Issues refer to all medicines supplied from hospital pharmacies: to wards; departments; clinics; theatres; satellite sites and to patients in outpatient clinics and on discharge 
organizations, and inevitably full implementation will need to take place over a number of years. Any individual guideline will show a mixed picture of implementation. For example, studies looking at the implementation of the head injury clinical guideline published in $2003^{8}$ demonstrate mixed progress, with the frequency and documentation of neurological observation as recommended by the guideline reported to be incomplete following guideline implementation in two local audits; $;{ }^{9} 10$ whereas Hassan and colleagues reported a two-fold increase in use of CT head scan rate (3\% to $7 \%$ ) following implementation of the guideline, with a corresponding decrease in the rate of standard X-ray (37\% to $4 \%$ ) and admission, in line with guidance recommendations. ${ }^{11}$

The putting into practice of national guidelines can be affected by various factors. Barriers can exist at a number of levels, from the national (including the regulatory environment), to the organizational (such as lack of staff, resources and managerial leadership), and the individual level. ${ }^{12}$ Particular factors affecting individuals include clinicians' concerns about guidance undermining their autonomy, ingrained practices that might be susceptible to change, and general disagreement with the guidance content.

There is no point in developing guidance for the NHS if it does not have a significant impact on patient care and outcomes. Three years ago, therefore, NICE launched a programme to support the implementation of its guidance. Mirroring the areas where barriers to implementation have been identified, this programme is designed to have an impact at national, organizational and individual levels.

Support at a national level is largely through the Healthcare Commission's inspection process against standards $;^{13}$ NICE technology appraisals and interventional procedures are defined as 'core standards' and clinical guidelines and public health guidance as 'developmental standards' in the Department of Health's document Standards for Better Health. ${ }^{14}$ This requires organizations to be able to demonstrate they are implementing technology appraisals within the three month funding directive ${ }^{15}$ (unless there is a published exception); compliant with interventional procedure guidance; and working towards achieving the standards set in NICE clinical and public health guidelines.

Many factors are known to influence successful implementation of guidance. Implementation is often reliant on enthusiastic clinicians driving forward change with the aim of ensuring their practice is based on the best available evidence. Clinicians are unlikely, however, to be able to drive forward change without organizational support. The key aspects of such support are outlined in How to Implement NICE Guidance, which describes the key organizational stages that would be expected in any successful programme of change. Five components are identified in the guide: board support and clear leadership; a dedicated resource (i.e. input from someone with ringfenced time); support from a relevant multi-professional group; a systematic approach to planning, including relevant financial aspects associated with any change; and finally a process for evaluating uptake. Examples of shared learning of effective processes of implementation incorporating these principles can be found on the NICE website (www. nice.org.uk), but support from relevant clinical groups remains essential. Local trusts therefore need to consider how to engage clinicians in any process of change, building on any involvement that may have occurred as part of the guideline development process.

\section{SUPPORTING IMPLEMENTATION ACTIVITY}

Research into effective means of changing clinical behaviour has examined a number of different approaches. Because of the complex nature of human behaviour, it is not surprising that no approach is effective alone and that a tailored, multifaceted approach is required. ${ }^{12}$ Some of the more effective methods include interactive educational meetings, educational outreach visits to practices (especially effective on prescribing habits), reminders (including computer-based decision support), and the use of clinical audit. To support these activities at a local level, NICE produces a range of materials through the NICE website (Box 2), including slide sets for use at educational meetings, programmes of selfdirected study through BMJ Learning and doctors.net.uk, and audit criteria. There is also a small team of implementation consultants with the capacity to support implementation at a local level.

It is important to be aware of the extent of and challenges to uptake of national guidance. It is not the role of NICE to monitor implementation (that is carried out by

Box 2 Examples of NICE Implementation Support Tools. (See www.nice.org.uk, under the heading 'Using Guidance')

- Forward planner: summarizing published guidance organizations may still be implementing and listing forthcoming guidance to help planning ahead

- National cost impact report and costing templates

- Implementation advice: provides practical suggestions on local implementation, includes a review of the policy context and the key drivers, and identifies the likely implementation barriers and possible ways to overcome these for different audiences

- Slide sets: provide key messages and a framework for discussion with different audiences

- Audit criteria

- Education tools: online learning packages with BMJ Learning and doctors.net.uk

- Commissioning guides: tools to suppoft evidence based commissioning in line with NICE guidance 
the Healthcare Commission and individual organizations); however, NICE has developed a database of guidancespecific NICE implementation uptake reports produced inhouse and references to external literature concerning uptake of guidance (Evaluation and Review of NICE Implementation Evidence [ERNIE]) and can be viewed on the NICE website under the heading 'Using Guidance'.

\section{CONCLUSIONS}

As research evidence and medical knowledge continue to expand, the use of national guidance will increasingly become an essential mechanism for informing day-to-day practice and keeping up to date with new information. Organizations like NICE will therefore have a responsibility for ensuring that guidance is kept current, is provided in an accessible format, and is available through a variety of different routes. Ensuring that the recommendations are integrated in electronic decision support systems will also be essential to informing practice in a practical way. Continuing to learn from research and practice about the levers supporting implementation - and the barriers impeding it - and further developing the support strategy will also be key. It is then the role of individual clinicians and organizations to review clinical practice and ensure that mechanisms are in place to be confident that the most clinical and cost effective care is being given.

Within the context of clinical governance, which makes individual professionals and organizations accountable for improving the quality and effectiveness of their services, and in the context of increasing demands on the health service with advances in technology, it is anticipated that individual professionals and organizations will continue to work towards full implementation of NICE guidance. We hope that our implementation work programme will continue to support implementation and ensure clinical care is based on the best possible evidence to increase health and well-being.

Competing interests All authors work for NICE.

Guarantor JC.
Contributorship JC and GL wrote the paper. TL analysed and presented the data.

\section{REFERENCES}

1 General Medical Council. Good Medical Practice. London: GMC, 2006. Available at www.gmc-uk.org/guidance

2 Breen A, Feder G. Where does the evidence come from? In: Hutchinson A, Baker R (eds). Making Use of Guidelines in Clinical Practice. Abingdon: Radcliffe Medical Press, 1999

3 Gaebel W, Weinmann S, Sartorius N, Rutz W, McIntyre JS. Schizophrenia practice guidelines: international survey and comparison. BrJ Psych 2005;187:248-55

4 Sheldon TA, Cullum N, Dawson D, et al. What's the evidence that NICE guidance has been implemented? Results from a national evaluation using time series analysis, audit of patients' notes, and interviews. BMJ 2004;329:999

5 Rawlins M, Culyer AJ. National Institute for Clinical Excellence and its value judgements. BMJ 2004;329:224-7

6 National Institute for Health and Clinical Excellence. Incorporating health economics in guidelines and assessing resource impact. In: The Guidelines Manual. London: NICE, 2007: chapter 8. Available at http://www.nice.org

7 Hanies A, Kuruvilla S, Borchert M. Bridging the implementation gap between knowledge and action for health, Bull WHO 2004;82:724-32

8 National Institute for Health and Clinical Excellence. Clinical Guideline 4. Head injury: Triage, Assessment, Investigation and Early Management of Head Injury in Infants, Children and Adults. London: NICE, 2003. Available at http://www.nice.org

9 Campbell A, Reilly D. Are head injury guidelines being met? Clin Gov 2005; 10:57-8

10 Hassan Z, Smith M, Littlewood S, et al. Head injuries: a study evaluating the impact of the NICE head injury guidelines. Emerg Med $J$ $2005 ; 22: 845-9$

11 Qureshi AA, Mulleady V, Patel A, Porter KM. Are we able to comply with the NICE head injury guidelines? Emerg Med J 2005;22:861-2

12 Grol R, Wensing $\mathrm{M}$, Eccles $\mathrm{M}$ (eds). Improving Patient Care. The Implementation of Change in Clinical Practice. Oxford: Elsevier, 2004

13 Healthcare Commission. Assessment for Improvement: The Annual Health Check 2005. London: Healthcare Commission, 2005. Available at http: / /www.healthcarecommission.org.uk

14 Department of Health. Standards for Better Health. London: DoH, 2004. Available at http://www.dh.gov.uk

15 Department of Health. Secretary of State's Direction: Directions to Primary Care Trusts and NHS trusts in England concerning Arrangements for the Funding of Technology Appraisal Guidance from the National Institute for Clinical Excellence (NICE). London: DoH, 1 July 2003. Available at http://www.dh.gov.uk/en/Publicationsandstatistics/Publications/ PublicationsLegislation/DH_4083088 